\title{
Micro-hole and strip plate-based photosensor
}

\author{
E.D.C. Freitas ${ }^{\text {a }}$, J.F.C.A. Veloso ${ }^{\text {b,* }}$, A. Breskin ${ }^{\text {c }}$, R. Chechik ${ }^{\text {c }}$ F.D. Amaro ${ }^{\text {a }}$, \\ L.F. Requicha Ferreira ${ }^{a}$, J.M. Maia ${ }^{a, d}$, J.M.F. dos Santos ${ }^{a}$ \\ ${ }^{a}$ Physics Department, University of Coimbra, 3004-516 Coimbra, Portugal \\ ${ }^{\mathrm{b}}$ Physics Department, University of Aveiro, 3810-193 Aveiro, Portugal \\ ${ }^{\mathrm{c}}$ Department of Particle Physics, The Weizmann Institute of Science, 76100 Rehovot, Israel \\ ${ }^{\mathrm{d}}$ Physics Department, University of Beira Interior, 6200-001 Covilhã, Portugal
}

Available online 16 May 2007

\begin{abstract}
The performance of a new VUV photosensor, based on an micro-hole and strip plate (MHSP) electron multiplier with a CsI photocathode deposited on its top electrode, is described. This photosensor presents gains above $10^{4}$ when operating in an Ar- $5 \% \mathrm{Xe}$ gas mixture at $1 \mathrm{~atm}$. Although the gain of a single MHSP is not sufficient for efficient detection of single photons, this simple UV photosensor may be useful for the detection of higher light levels, such as primary- and secondary scintillation in noble gases.

(C) 2007 Elsevier B.V. All rights reserved.
\end{abstract}

PACS: 29.40.Cs; 29.40.-n; 85.60.Gz

Keywords: MHSP; CsI photocathode; Noble gas scintillation

\section{Introduction}

The micro-hole and strip plate (MHSP) [1] is a rather new gaseous electron multiplier conceived as a combination of a Micro-strip plate (MSGC) and a gas electron multiplier (GEM) in a single-, double-sided element. It integrates, on a single plate, two successive independent stages of charge amplification, a GEM-like hole avalanche and an MSGC-like anode-strip avalanche. Like the GEM, the MHSP is fabricated with printed circuit board technology from a $50-\mu \mathrm{m}$ Kapton foil, metalized with 5 - $\mu$ m-thick copper layers on both sides. A GEM-like pattern of holes is etched through the foil with a continuous metal electrode on the top side, and a standard micro-strip pattern etched on the bottom side, with the holes centered on the wide cathode strips.

The MHSP presents a high total gain; it also optically decouples the anode-strip charge multiplication region from the electron drift region, reducing photon feedback effects. In addition, it has highly reduced ion backflow,

\footnotetext{
*Corresponding author. Tel: ++351239410667 ; fax: ++351239829158 .

E-mail address: jveloso@fis.ua.pt (J.F.C.A. Veloso).
}

e.g., in a single MHSP more than $80 \%$ of the avalanche ions are trapped on the nearby cathodes [2]; this helps controlling ion-induced feedback effects.

In this work, we report on the performance of a photosensor based on an MHSP with a reflective CsI photocathode deposited on its top side (Fig. 2), similar to GEM-based photon detectors [3,4]. An attractive feature is the concept of a windowless photosensor, in which both scintillator and photosensor operate within the same gas volume.

Several concepts of gaseous photosensors incorporating CsI photocathodes, suited for the detection of scintillation light, have been described in the literature. Among them are avalanche- and wire-chambers [5], multi-GEM [4,6] and multi-GEM and MHSP detectors [2], capillary-plate detectors [7], and, more recently, thick GEM-like devices [8]. This new type of photosensor combines a simple design with high-gain capability, even at high pressure $[1,9]$.

\section{Experimental system}

The photosensor's performance was investigated in combination with a gas proportional scintillation counter 
(GPSC), both placed within a vessel filled with $\mathrm{Ar}-5 \% \mathrm{Xe}$ at $1 \mathrm{~atm}$; the photosensor was placed at a distance of $3 \mathrm{~mm}$ from the scintillation region (Fig. 1). X-rays $(5.9 \mathrm{keV})$ interact in the GPSC's absorption/drift region, inducing primary-electron clouds, which drift toward the $1.5-\mathrm{cm}$ thick scintillation region. The intensity of the electric field in the scintillation region, between the meshes G1 and G2, was chosen such that the primary electrons gain sufficient energy to excite, but not ionize, the gas atoms; the latter resulted in the emission of secondary scintillation (a narrow continuum, $\sim 10 \mathrm{~nm}$ FWHM, peaking at $172 \mathrm{~nm})$.

The CsI-MHSP photosensor operation principle is presented in Fig. 1. The forward-emitted VUV light from the scintillation region is converted in the CsI photocathode, producing photoelectrons that are extracted and focused into the MHSP holes and undergo double-stage multiplication with final avalanche at the anode strips in the MHSP bottom side. The avalanche ions are mostly collected on the cathode plane and cathode strips. The final avalanche at the anode strips is fully concealed from the CsI photocathode, preventing any secondary-photon feedback. This characteristic is very important in pure noble gases, with high VUV-photon transparency.

The MHSP used in our tests has an active area of $2.8 \times 2.8 \mathrm{~cm}^{2}$. The holes are bi-conical, around $\varnothing 40 / 70 \mu \mathrm{m}$ in the Kapton/copper film; they are arranged in an asymmetric hexagonal lattice of $140-$ and $200-\mu \mathrm{m}$ pitch in the directions parallel and perpendicular to the strips, respectively. The anode and cathode micro-strip widths are 30 and $100 \mu \mathrm{m}$, respectively, with a $35-\mu \mathrm{m}$ gap between them and a $200-\mu \mathrm{m}$ pitch. All the MHSP electrodes are independently polarized, with the top electrode at ground potential. The dipole (GEM-like) electric field within the holes is established through a potential difference between the cathode strips and the top electrode, $V_{\mathrm{CT}}$, while the potential difference between the anode and the cathode

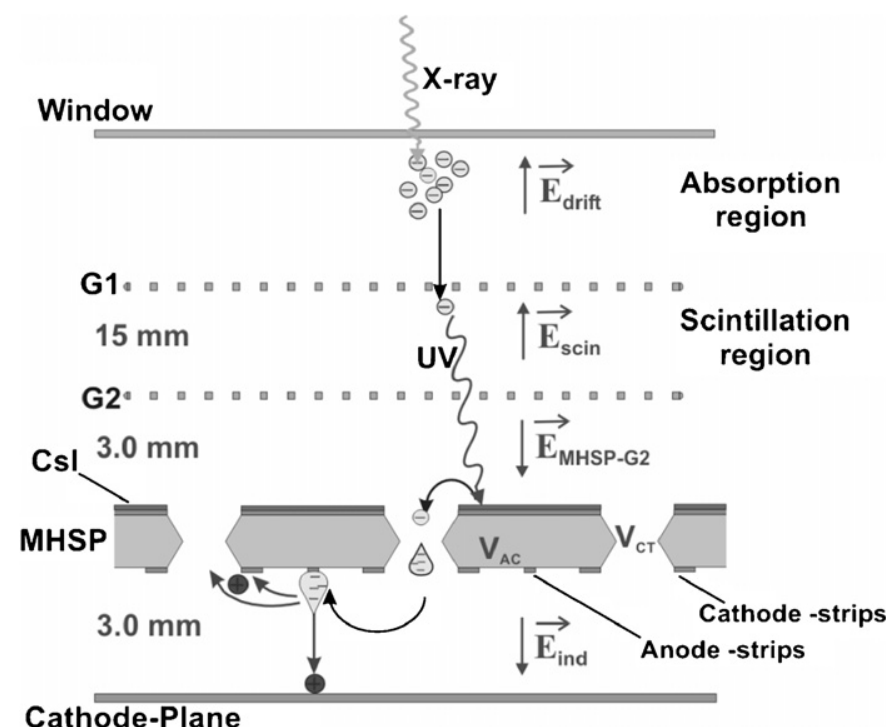

Fig. 1. Schematic diagram of the GPSC with the CsI-MHSP photosensor. strips, $V_{\mathrm{AC}}$, establishes an MSGC-like electric field on the bottom side of the MHSP. The bottom mesh electrode was operated at negative voltage to capture as much as $50 \%$ of the positive ions produced in the final avalanche around the anode strips [4].

Detector signals from the anode strips were fed through a Canberra 2006 charge preamplifier (sensitivity of $1.5 \mathrm{~V} /$ pC) and a Tennelec TC243 linear amplifier (4- $\mu$ s shaping time) to a multi-channel analyzer. The electronic chain sensitivity was calibrated for absolute gain measurement, using a calibrated capacitor directly connected to the preamplifier input and to a precision pulse generator.

\section{Experimental results and discussion}

An electric field, for the MHSP-G2 gap, with opposite direction of that presented in Fig. 1 provides means to separately accumulate the primary-charge signals and the scintillation signals, and study the relation between them. In Fig. 2 we present a pulse-height distribution recorded with $5.9-\mathrm{keV} \mathrm{X}$-rays and an electric field of $3.7 \mathrm{kV} / \mathrm{cm}$ in the GPSC scintillation region. In this measurement, an electric field of $100 \mathrm{~V} / \mathrm{cm}$ was applied to the MHSP-G2 gap, with opposite direction of that presented in Fig. 1. In these conditions, the electrons traversing the scintillation region are still collected at $\mathrm{G} 2$, but primary electrons resulting from $5.9 \mathrm{keV}$ X-ray interactions in the MHSP-G2 gap are directed toward the MHSP, producing the peak marked in the low-energy region of the spectrum. These events have amplitudes that are independent of the scintillation region bias and are detected even without the presence of secondary scintillation. On the other hand, they disappear from the spectrum when the electric field in the MHSP-G2 region is slightly reversed, as depicted in Fig. 1. We assume that these electrons, originated from X-ray conversion in the MHSP-G2 gap, undergo a similar MHSP

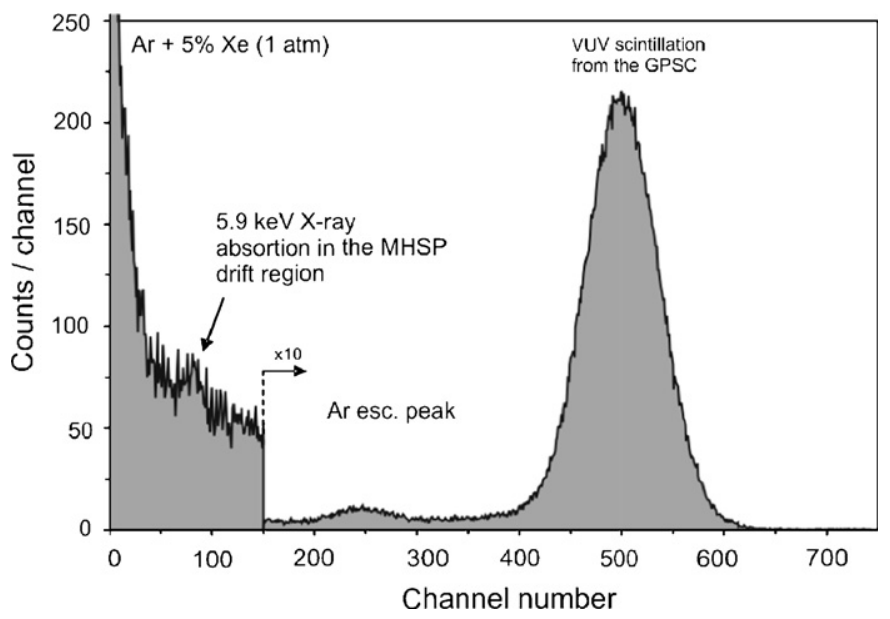

Fig. 2. Typical pulse-height distribution induced by $5.9-\mathrm{keV}$ X-ray interactions in the GPSC, recorded from the CsI-based MHSP photosensor with a positively biased MHSP-G2 gap. 
gas multiplication as photoelectrons produced in the CsI photocathode. Therefore, the ratio of the scintillation peak, which is proportional to the average number of photoelectrons produced by each X-ray, and the direct conversion peak, which is proportional to the number of ionization electrons produced by each X-ray, provides the scintillation gain, $L$. A value of $L \sim 6$ is obtained from Fig. 2 . This value is consistent with that obtained for a GPSC based on a CsIMSGC photosensor operated with $\mathrm{Ar}-5 \% \mathrm{Xe}$ gas mixture (Fig. 5 of Ref. [12]).

For constant biasing conditions in the GPSC drift and scintillation regions, $L$ is proportional to the photoelectron collection efficiency, $C_{e}$, in the photosensor. We have studied the relative behavior of $C_{e}$ as a function of the field between the MHSP and G2, Fig. 3; the drift and scintillation fields were kept constant at $E_{\text {drift }}=0.5$ and $E_{\text {scint }}=3.1 \mathrm{kV} / \mathrm{cm}$, while $V_{\mathrm{CT}}$ and $V_{\mathrm{AC}}$ were varied. As in other works $[10,11]$, the data show a relative behavior that is almost independent of the MHSP biasing conditions; the highest photoelectron collection efficiency is obtained for slightly reversed electric fields across the MHSP-G2 gap of $\sim-75 \mathrm{~V} / \mathrm{cm}$, which is probably the value required to cancel out the field penetration from the scintillation region. At higher reversed-field values, a higher fraction of photoelectrons are collected on G2, naturally on the account of those directed into the holes. At normal field direction, the number of photoelectrons directed into the holes decreases due to the decrease of photoelectron extraction from the photocathode [10]. A slightly reversed field above the photocathode, Fig. 1, does not seriously affect the photoelectron collection efficiency, but removes from this region unwanted radiation-induced electrons.

Fig. 4 presents the CsI-MHSP photosensor gain as a function of $V_{\mathrm{AC}}$, for $V_{\mathrm{CT}}=250 \mathrm{~V}$ (Fig. 4a) and as a function of $V_{\mathrm{CT}}$, for $V_{\mathrm{AC}}$ voltages of 130 and $180 \mathrm{~V}$ (Fig. 4b). This gain was obtained dividing the detector total gain by the value obtained for $L$, at each point. As expected, the experimental data reveal a characteristic exponential

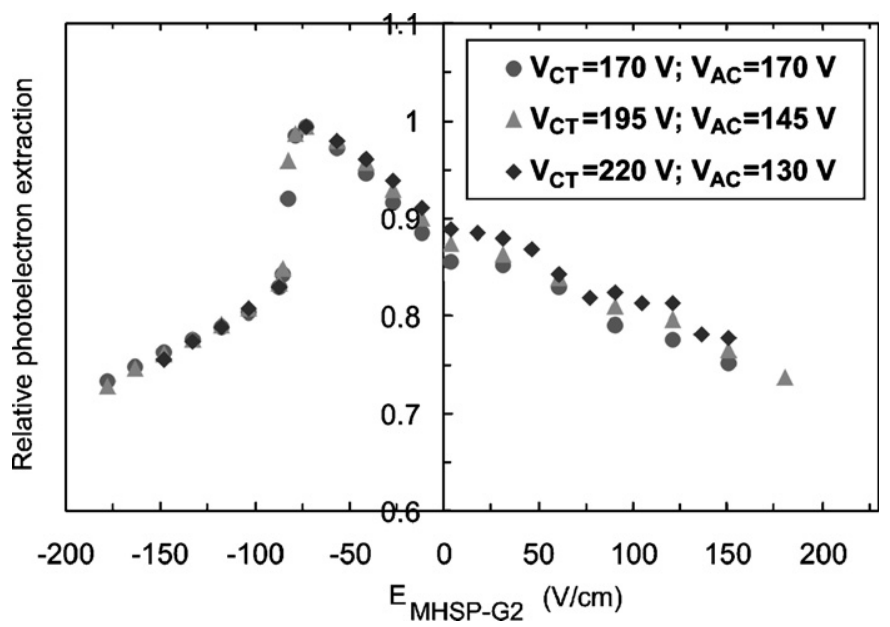

Fig. 3. Detector relative amplitude as a function of the electric field intensity in the MHSP-G2 gap for different biasing conditions of $V_{\mathrm{AC}}$ and $V_{\mathrm{CT}}$.
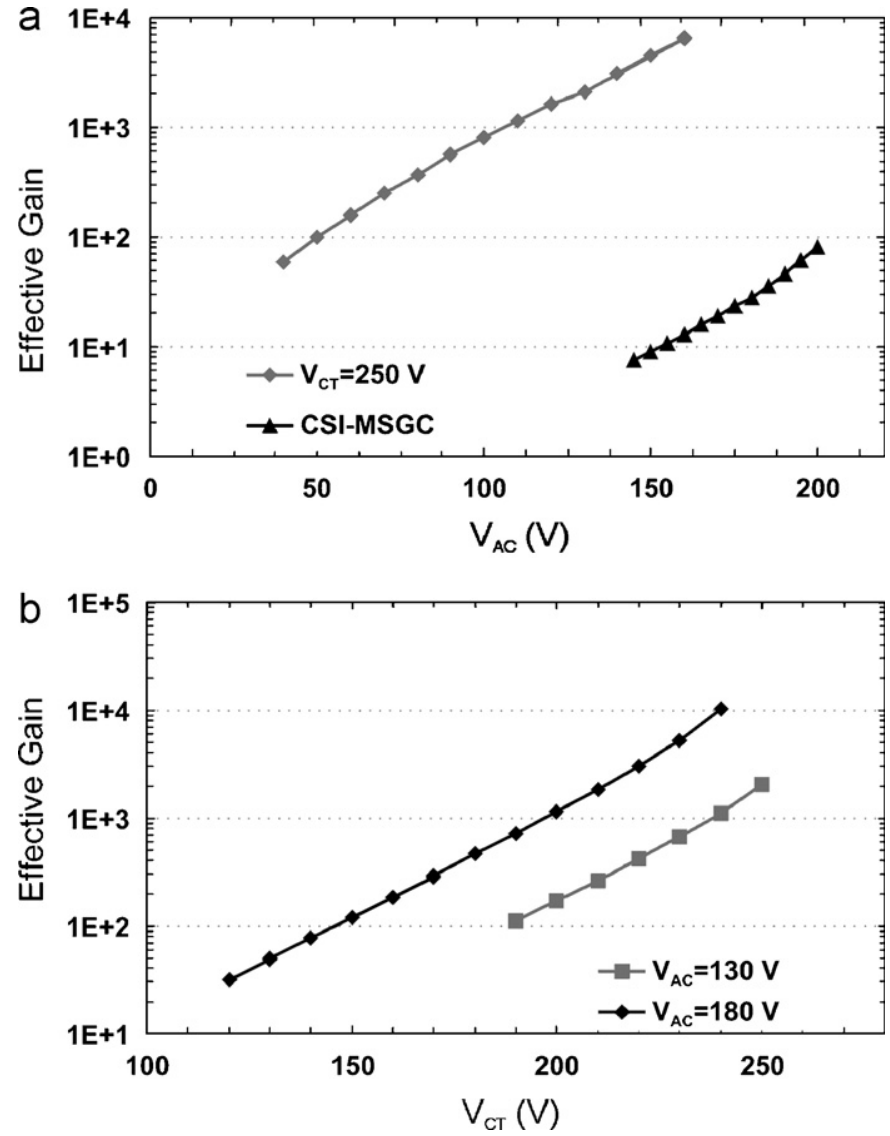

Fig. 4. CsI-MHSP photosensor gain as a function of: (a) $V_{\mathrm{AC}}$, for $V_{\mathrm{CT}}=250 \mathrm{~V}$; (b) $V_{\mathrm{CT}}$, for $V_{\mathrm{AC}}$ of 130 and $180 \mathrm{~V}$; for $\mathrm{Ar}-5 \% \mathrm{Xe}$ gas at $1 \mathrm{~atm}$. The gain obtained for the CsI-MSGC photosensor [12] is also presented in (a), for comparison.

dependence of the charge avalanche process in the proportional regime. Gains above $10^{4}$ were reached without discharges, in consistence with the results obtained with a MHSP operated in the same gas mixture [1]. The CsI-MHSP photosensor reaches higher gains and lower detection limits, two orders of magnitude better than for a CsI-MSGC photosensor operated in an $\mathrm{Ar}-5 \% \mathrm{Xe}$ GPSC [12], free of any photon feedback effects. On the other hand, similar or higher gains can also be achieved with multi-GEM detectors that allow suppressing the ion backflow as well, e.g., Ref. [4].

\section{Conclusions}

We have studied an MHSP electron multiplier with a CsI photocathode deposited on its top electrode, operating in an $\mathrm{Ar}-5 \% \mathrm{Xe}$ gas mixture at $1 \mathrm{~atm}$. This simple photodetector can provide gains above $10^{4}$ in a single element, two orders of magnitude higher than that achieved for the $\mathrm{Ar}-5 \% \mathrm{Xe}$ CsI-MSGC photosensor. Although the gain achieved with a single MHSP is not sufficiently high for single photon detection, this simple UV photodetector may be useful for the detection of higher light levels, as for 
example the radiation-induced primary- and secondary scintillation of noble gases.

Future work will include the study of similar photosensors in pure Xe for higher filling pressures.

\section{Acknowledgments}

This work was supported by Project POCTI/FP/FNU/ 50218/03, FEDER and FCT (Lisbon), and by the Israel Science Foundation Project 151/01. A. Breskin is the W.P. Reuther Professor of Research in peaceful use of atomic energy.

\section{References}

[1] J.F.C.A. Veloso, et al., Nucl. Instr. and Meth. A 524 (2004) 124.

[2] J.M. Maia, et al., Nucl. Instr. and Meth. A 523 (2004) 334.

[3] R. Chechik, et al., Nucl. Instr. and Meth. A 502 (2003) 19.

[4] T. Meinschad, et al., Nucl. Instr. and Meth. A 535 (2004) 324.

[5] F. Piuz, Nucl. Instr. and Meth. A 502 (2003) 76.

[6] D. Mörmann, et al., Nucl. Instr. and Meth. A 504 (2003) 93.

[7] C. Iacobeus, et al., Nucl. Instr. and Meth. A 525 (2004) 42.

[8] R. Chechik, et al., Nucl. Instr. and Meth. A 553 (2005) 35.

[9] F.D. Amaro, et al., J. Inst. 1 (2006) P04003.

[10] D. Mörmann, et al., Nucl. Instr. and Meth. A 530 (2004) 258.

[11] Z. Frankel, et al., Nucl. Instr. and Meth. A 546 (2005) 466.

[12] C.M.B. Monteiro, et al., IEEE Trans. Nucl. Sci. NS-49 (2002) 907. 